\title{
PE Teaching Model in Secondary Schools of the United States and Japan and Its Enlightenment
}

\author{
Yingdong SONG \\ Jingdezhen Ceramic Institute, Jingdezhen, 333403, China \\ syingdong@126.com
}

\begin{abstract}
Through a systemic introduction of PE teaching model in the United States and Japan, and combined with the issues existing in the construction of secondary school PE teaching model in China, this research suggests that the PE teaching model in secondary schools of China should establish a clear and consistent PE teaching model philosophy; construct diverse and innovative PE teaching models; enrich teaching designs.
\end{abstract}

KEYWORD: Secondary school; PE teaching model; Enlightenment

\section{INTRODUCTION}

What is "PE teaching model"? At present, the perspective of Professor Mao Zhenming is more representative currently, namely, the "PE teaching model" is a teaching procedure that embodies some sort of teaching ideas. It includes relatively stable procedure structure of teaching and corresponding teaching method system, which is mainly manifested in the teaching unit as well as the design and implementation of teaching courses. And it is believed that PE teaching model is a mature and stable PE teaching procedure structure in essence, and the formation of PE teaching procedure structure reflects the characteristics of the basic laws of some sort of teaching procedure."

\section{SURVEY OF RESEARCHES ON PE TEACHING MODEL AT HOME AND ABROAD}

Research on PE teaching model is the choice, abstraction and generalization of various effective ways of PE teaching activities, which is the intermediary between the practice and theory of PE teaching, and the acceptance of this kind of intermediary liability determines that the research on PE teaching model not only deepens the teaching practice, but also plays a very important role in the construction of teaching theory. The teaching model began to be discussed extensively in 1972 with the first edition of Model of Teaching of Joyce and Wale who come from the United States. In the early 80s, the educational theory circle researched on the teaching model for the first time along with the publication of $\mathrm{Wu}$ Guangpu's "exploration of teaching model". Research on PE teaching model began to rise in the range of PE teaching theory from the late $80 \mathrm{~s}$. There were both practical exploration and theoretical considerations. In 1998, Liu Shao wrote a comprehensive introduction to a dozen kinds of teaching methods, and the wording meaning of the teaching methods is basically the same as the teaching model. The theoretical research articles of PE teaching model have gradually increased since then. In 1998, Mao Zhenming made "theory of PE teaching model" in PE Science with the discussion of the concept and classification of PE teaching model. Zhao Li and Yang Tieli also carried out indepth researches on PE teaching model, and conducted classification for PE teaching model according to the view of teaching model classification of the scholar Hirooka sukezox who comes from Japan. Zhong Bingshu who comes from Beijing Sport University conducted a research on volleyball teaching by using programmed teaching model; Li Jiekai comes from Shenyang Sport University carried out a research on fundamental basketball teaching by using discovery teaching model. The current researches on PE teaching model in China mainly focus on three aspects: first of all, research on theoretical aspects, such as the conception, structure, theory of teaching model; secondly, the test promotion of successful PE teaching models at home; thirdly, the improvement of teaching method and the optimization of teaching organization forms. 


\section{THE MAJOR ISSUES EXISTING IN SECONDARY SCHOOL PE TEACHING MODEL IN CHINA}

The construction of a scientific and effective PE teaching model has a growing importance for promoting the healthy development of $\mathrm{PE}$ with the continuously deepening of $\mathrm{PE}$ teaching reform in China. The PE teaching at secondary school stage is a key stage for students to become interested in physical training, as well as cultivating their physical habits. At present, there are several shortcomings existing in the PE teaching model of secondary school in China: first of all, the goal of teaching model is single. The classroom teaching model which gives priority to the impartment of motor skill in China ignoring the development of student's personality, physical ability and attitude, which is difficult to meet the needs of the development of the society and students. Secondly, the concept is disjointed with practice. The PE teaching goal has new changes compared with what it used to be along with the proposing of new PE courses concept. However, we did not create new teaching model to coincide with it. It is impossible for single model structure to both complete the goal of knowledge and skills as well as the teaching goal of emotions, personality and behaviors, which resulted in the separation between teaching philosophy and teaching practice; thirdly, the operation of teaching model is poor. There were numerous new teaching models have been proposed in China, but most of these models are unable to be used effectively in practice, and the applicable and achievable goals are very simple, which is related to the imperfection of the theory structure of these models, and the lack of specific teaching design as well as the scientific evaluation means.

Schools in the United States and Japan have rich practice experiences in PE teaching because they carried it out earlier. Stones from other hills may serve to polish the jade of this one. Research on the PE teaching model of the United States and Japan is contribute to examining the issues existing in secondary school physical education in China, and by absorbing the advanced ideas of PE teaching model in the United States and Japan to put forward suggestions for the reform and development of PE teaching model in China, so as to work out practical solutions.

\section{OVERVIEW OF THE PE TEACHING MODEL IN THE UNITED STATES}

\subsection{Themes}

Nowadays, the goal of physical education has different expressions in each State of the United States with a common core of idea that is to improve the health of students. Health is the most important in their opinion, so the main goal of PE curriculum is for the health of each student. Scholars have built a wide variety of PE teaching models under the guidance of these thoughts.

\subsection{Main types and characteristics}

There are four main representative PE teaching models atsecondary school stage of the United States: multi-activity-based model, sport education model, fitness education model and social development model.

\subsection{1 multi-activity-based model}

Multi-activity-based model is the most common teaching model in primary and secondary schools of the United States, which takes physical activities as the curriculum core, and focuses on the learning of skills, so as to maintain the diversification of activities and introduce the skills of a variety of activities. Multi-activity-based model has the characteristics of diversification and joy which meet the needs of students. It gives students the opportunity to explore and experience more physical activities, so as to help students gain a variety of skills, so that the students are able to expertly participate in various activities once they enter adulthood. Multi-activity-based model learns a wide variety of activities in a short unit; as a result, some scholars believe that the multi-activity-based model does not have adequate time to develop the physical abilities, the mastery of action skills and knowledge of the students.

\subsubsection{Sport education model}

Sport education model is first proposed by Siedentop (1994) who comes from The Ohio State University at Columbus, which is a kind of way based on activity to train students just like real athletes in all aspects. It focuses on technology, rules, tactics, appreciation and sportsmanship with three main goals. The first one is to teach students motor techniques and tactics in order to increase students' physical qualities; the second one is to enable students to participate in PE activities, quickly becoming a team member so as to participate in PE competitions; the third one is making students act as multiple roles such as coaches, record specialists, statisticians, equipment manager, and referees and so on, so as to be able to plan and manage a competitive PE activity.

\subsubsection{Fitness education model}

Fitness education model is advocated by Cordin and Lindsey (1996), which not only pays attention to enable students to participate in PE activities, but also focuses on developing the students' concept of lifelong participation in physical activities. It promotes health through PE activities, and educates 
students to have a positive attitude for PE activities. It focuses on the elucidation, experiments and the preparation of appropriate exercise plans with regard to teaching, so as to help students learn about healthrelated activities, thereby enabling students to become good designers of physical fitness and problem-solvers. Fitness physical education model pays attention to impart fitness knowledge and concept to students. The research finds that fitness physical education model is a kind of PE teaching model that can effectively promote students' physical knowledge and cultivate students' consciousness of lifelong sports. The shortcoming of this model is that it takes more time to explain and analyze, and there is relatively little time for physical skills learning.

\subsubsection{Social development model}

Social development model is put forward by HELLISON (1985, 1995), which is designed to develop students' sense of responsibility for individual and society. It mainly emphasizes the social skill of students, and the self-control behaviors. The social skill is divided into six levels by HELLISON to enable students to gradually improve and encourage students to work out their scores in each tier, and then compared with the scores evaluated by teachers. The teachers and students check the ideas, as well as discussing the situation of the progress. Social development teaching model is to educate students to sacrifice themselves and understand others by PE activities firstly; secondly, it allows students to participate in $\mathrm{PE}$ activities and try with their best efforts; thirdly, it improves the self-direction of students, enabling them to understand from their heart of how to participate and how to regulate their own behaviors; fourthly, it enables students to get out of themselves, and for the sake of others; finally, the students can help other students regulate the behaviors in physical education class.

\section{OVERVIEW OF THE PE TEACHING MODEL IN JAPAN}

\subsection{Themes}

Japan takes the accomplishment of cultivating "democratic population with healthy mind and body" as the mission, advocating lifelong sports. To this end, the PE course in the school education subjects is called "health physical course". The PE course in Japan adheres to the internal unity of the motivation of personal participation in sports and the social value of PE disciplines with full respect for the selfregulated study and individual differences of students, as well as paying attention to the experience of students for the fun of sports, including the understanding of the sports significance. In terms of specific teaching design, it emphasizes on understanding the connotation of sports culture by the subjective and exploratory motor technique learning of students.

\subsection{Main types and characteristics}

\subsection{The teaching model of "happy sports"}

The teaching model of "happy sports" in Japan is guided by the thought of "lifelong sports", which requires the sports teaching cannot have mandatory but respect students' spontaneity, autonomy, selfdiscipline and individual differences, so as to enable them to gain fun from the physical education, as well as fully playing their existing capacity to enjoy sports, deepening their understanding and sentiment for sports. Improve student's ability of enjoying the fun of sports in order to lay a foundation for their lifelong sports. It emphasizes on learning while taking teaching as a supplement in happy sport learning. After students selecting the projects based on their interests, the teachers will provide technical guidance theoretically, and then the students establish the appropriate learning goals according to the suggestive learning contents from the teachers and their ability, and enjoy the fun of sports by exercises and competition, thereby developing their sports skills.

\subsection{2 "Discovery-style" teaching model}

The "discovery-style" teaching model of Japan is a kind of teaching model which takes "discoverymethod" as the main and is advocated actively in Japan currently. It aims to cultivate the innovative spirit and ability of students, so as to fully reflect the effects of sports on the cultivation of personality. It advocates the unity of understanding and mastery, enabling students to master the motor technique in understanding, so the teaching process is designed and implemented according to the general cognitive principles, which requires students with strong study skills and independent learning skills, including a deep knowledge and culture background of the teachers who have strong PE teaching ability and are good at flexibly using heuristic teaching.

\section{THE ENLIGHTENMENT OF PE TEACHING MODEL IN THE UNITED STATES AND JAPAN FOR THE PHYSICAL EDUCATION IN SECONDARY SCHOOL OF CHINA}

\subsection{Establish clear and consistent PE teaching philosophy}

We can clearly get the respective philosophies from the PE teaching model of the United States, and it can also be seen from their respective names. For example, multi-activity-based model emphasizes the learning of skills, so as to maintain the diversification of activities and introduce the skills 
of a verity of activities; while the social development model is designed to develop students' sense of responsibility for individual and society, which envisages to shape the emotions and qualities of the younger generation through PE curriculum, such as clear self-awareness, self-judgment, selfunderstanding and personal relationships, cooperation, sharing, tolerance and so on. The physical education in China should absorb the teaching experience of school physical education in the United States and Japan, so as to clear-cut propose the teaching philosophy of physical education and health curriculum, and make it as a barometer for daily physical education which cannot be changed frequently, or it will difficult to adapt to in the teaching design.

\subsection{Construct diverse and innovative PE teaching model}

China has a vast territory and various minorities with an uneven regional development. The PE health curriculum in secondary school is national curriculum, but that does not mean a national unity of teaching model. It should learn from the diversity characteristic of PE curriculum model in the United States, and build diversified teaching models under the guidance of the overall teaching goal, especially the PE teaching model in line with the respective national characteristics and regional culture characteristics. The provincial Education Administration Department can formulate the plan of national curriculum implemented in the province (autonomous regions and municipalities) in accordance with the administration policies of the national curriculum and the local realities, planning local curriculum, and report it to Ministry of Education for the record, as well as organizing the implementation. Schools should depend on the specific circumstances of the local social and economic development, and combine with the tradition and advantages of the school to develop or choose their own model of innovative PE teaching model with regard to the specific interests and preferences of students.

\subsection{Enrich the teaching design}

Studies have shown that, the stage of secondary school is an important stage for developing interests in sports and sports habits, and a wealth of physical education designs can meet the diverse needs of students, thereby stimulating their interest in sports, as well as cultivating their exercise habits. It can learn from the diversified PE teaching models of the United States, and to flexible use them depending on the actual needs of teaching. For instance, the concept model of "happy sport" of Japan can be referred to while arranging specific sports technique teaching, using the multi-activity-based model of the
United States to allow students to active to participate in, and then use sport education model to design the lesson units into season when the motor skill of students has been improved, dividing students into several groups to carry out competitions, and allow students to play a variety of roles in the competition. The social development model can be taken into account while developing the interpersonal relationships of students and improving the ability of social adaptation.

\section{CONCLUSION}

The physical education at secondary school stage is a critical stage for developing the consciousness of lifelong sports and the habit of students. However, the PE teaching model in China has been taking the mastery of the action skills acquisition as the core for a long time, which limits the development of model to diversity and richness, leading to the cultivation of physical education ability and lifelong physical ability is ignored in the PE teaching at middle school stage. It should absorb and refer to the content of PE teaching model in middle schools of the United States and Japan, so as to guide the concept clearly and breakthrough the single teaching model of imparting action skills, designing diverse and novel PE teaching model according to the actual needs. Take the various hobbies and interests of students, the development of physical education ability, and active participation as an axis to organize teaching contents and design teaching models, so as to establish practical $\mathrm{PE}$ teaching models adapting to different parts of China.

\section{REFERENCES}

[1] Qu Zonghu \& Yang Wenxuan, extraterritorial school PE faxes. Beijing: The People's Sports Publishing House, 1999

[2] Zhang Hongtan, research on PE teaching model. Sports Science and Technology, 1996, (6)

[3] Wu Tao \& Hu Lijun, Comparative research of PE teaching model in the elementary and secondary schools of China, the United States, Japan and Germany Journal of Tianjin Institute of Physical Education, 1994, (3):51-56

[4] Pan Lingyun \& Wang Jian, Comparative analysis of contemporary PE teaching model at home and abroadand discussion on the shortcomings of the construction of contemporary PE teaching model in China. Journal of Shandong Institute of Physical Education and Sports, 2001, (4):53-55

[5] Wu Tao, analysis of PE teaching model in primary and middle schools of Japan. Hubei sports science,1990, (4): 69-70

[6] Liu Haiyuan, the enlightenment of PE curriculum model in the United States on the physical education and health curriculum reform in China. Journal of Xi'an Physical Education University 2008, (1): 106-109

[7] Cao Mingliang \& QuGuangwei, Overview of PE educational model in the United States. Journal of PLA Institute of Physical Education 2004, (4): 126-128 